\title{
Editorial
}

\section{Nonlinear Time Series 2013}

\author{
Ming Li, ${ }^{1}$ Carlo Cattani, ${ }^{2}$ S. C. Lim, ${ }^{3}$ and Massimo Scalia ${ }^{4}$ \\ ${ }^{1}$ School of Information Science \& Technology, East China Normal University, Shanghai 200241, China \\ ${ }^{2}$ Department of Mathematics, University of Salerno, Via Ponte Don Melillo, 84084 Fisciano, Italy \\ ${ }^{3}$ Faculty of Engineering, Multimedia University, 63100 Cyberjaya, Selangor, Malaysia \\ ${ }^{4}$ CIRPS, University of Rome, "La Sapienza", Piazza San Pietro in Vincoli 10, 00184 Rome, Italy
}

Correspondence should be addressed to Ming Li; ming_lihk@yahoo.com

Received 12 December 2013; Accepted 12 December 2013; Published 12 January 2014

Copyright (C) 2014 Ming Li et al. This is an open access article distributed under the Creative Commons Attribution License, which permits unrestricted use, distribution, and reproduction in any medium, provided the original work is properly cited.

Nonlinear time series plays a fundamental role in various fields of sciences and engineering, ranging from physical and life sciences to telecommunication engineering.

One of the most challenging tasks of research is to face nonlinear problems which nonlinearly depend on a large number of parameters and/or are based on unpredictable, random, or fractal-like behavior. Some of the more intriguing and modern topics such as telecommunication, traffic dynamics, complex networks, optimization, and fractional dynamics, can be described by nonlinear time series. However, the extraction of useful information from the nonlinear time series by the commonly used analytical techniques is often difficult due the presence of noise in the signals. In this issue, we have selected papers on the main theme of identifying correct methods for the optimal understanding of the relevant information hidden in a signal representing a nonlinear phenomenon. We believe that there is a strong interest on this topic, although a widely accepted methodology for a proper investigation of nonlinear time series is still an open question. Through the collected papers of this issue we want to illustrate some of the interesting approaches and smart techniques which we hope can be regarded as a small step towards a systematic method for analyzing nonlinear time series.

This special issue collects 16 papers with respect to nonlinear time series, its computations, and applications.

M. Li and W. Zhao's paper entitled "Convergence of sample autocorrelation of long-range dependent traffic" exhibits a radical property of teletraffic time series. The paper "Identification of nonstandard multifractional Brownian motions under white noise by multiscale local variations of its sample paths" by K. Ahn and I. Lee provides stable and simultaneous estimators of two parameters for nonstandard multifractional Brownian motions under white noise. M. Li's paper "On the long-range dependence of fractional Brownian motion" clarifies that fractional Brownian motion is long-range dependent on the Hurst parameter $0<H<1$. The paper by $\mathrm{Li}$ and Zhao, entitled "Wild fluctuations of random functions with the Pareto distribution," introduces the terms wild and wildest fluctuations of time series that follow heavy-tailed distributions, such as the Pareto distribution.

Peng et al.s paper "Topology identification of complex network via chaotic ant swarm algorithm" studies the issue of topology identification from the point of view of parameter optimization for complex networks. It is a pioneering work revealing an important phenomenon of Lorenz chaotic equation that was used in their simulation of the $i$ th node from a view of the golden ratio with respect to chaotic ant swarm algorithm. M. Pinchas' two papers, entitled "Symbol error rate as a function of the residual ISI obtained by blind adaptive equalizers for the SIMO and fractional Gaussian noise case" and "Residual ISI obtained by nonblind adaptive equalizers and fractional noise," provide examples of applying fractional Gaussian noise to communication engineering. These two papers provide of a set of theories of adaptive equalizers essential to communication systems. The paper "Adaptive synchronization of complex dynamical multilinks 
networks with similar nodes" by W. Wang et al. proposes a novel method of adaptive synchronization to describe the similarity of similar nodes in complex multilinks networks. The paper entitled " $A$ reconfigurable logic cell based on a simple dynamical system" by L. Li et al. introduces a new scheme to achieve a dynamic logic gate that can be flexibly varied to obtain different logic functions by adjusting specific parameters of a dynamical system, discovering that it is resistant to system noise. In addition, the paper contributes a significant result in electronics engineering that the system can be considered as a leaky integrator.

O. C. Yolcu's paper entitled "A hybrid fuzzy time series approach based on fuzzy clustering and artificial neural network with single multiplicative neuron model" studies fuzzy time series forecasting by using artificial neural networks with single multiplicative neuron model in the identification of fuzzy relation such that the architecture selection problem can be eliminated without the need for defuzzification step by constituting target values from real observations of time series. The paper " $A n$ ARMA type fuzzy time series forecasting method based on particle swarm optimization" by E. Egrioglu et al. discusses fuzzy time series forecasting. Its novelty in time series prediction is in presenting a novel first-order fuzzy time series by taking into account both autoregressive and moving average structures. Its practical significance is that the proposed model is time invariant. The paper by C. Kocak "First-order ARMA type fuzzy time series method based on fuzzy logic relation tables" attempts to carry out fuzzy time series prediction a step further by considering autoregressive moving average ARMA $(1,1)$ in order to eliminate the deficiency of conventional fuzzy time series predictors. The paper "Set pair analysis based on phase space reconstruction model and its application in forecasting extreme temperature" by Y. Zhang et al. establishes a forecasting approach called set pair analysis based on phase space reconstruction model to improve forecasting precision of a time series.

Z. Chen et al. in their paper "Sensor scheduling with intelligent optimization algorithm based on quantum theory" proposes an improved particle swarm optimization (PSO) algorithm using a best dimension mutation technique. $\mathrm{M}$. Carlini et al.s paper "Italian residential buildings: economic assessments for biomass boilers plants" deals with the economic feasibility of biomass boiler plants with specific regard to an existing residential building. It further investigates an Italian case that focuses on the attention on European and national regulations on energy efficiency and considers the recent public incentives and supporting measures. The paper by S. Wanqing et al., "Tool wear detection using Lipschitz exponent and harmonic wavelet," explains singularity analysis with harmonic wavelet for data processing.

\section{Acknowledgments}

We are grateful to all the authors of this special issue for their contributions and to the reviewers for their valuable comments. Ming Li acknowledges the supports for his work in part by Macau Science and Technology Development Fund under Grant nos. 061/2011/A3, 009/2010/A1, and 021/2009/A1 and by the National Natural Science Foundation of China under the Project Grant nos. 61272402, 61070214, and 60873264 .

\author{
Ming Li \\ Carlo Cattani \\ S. C. Lim \\ Massimo Scalia
}




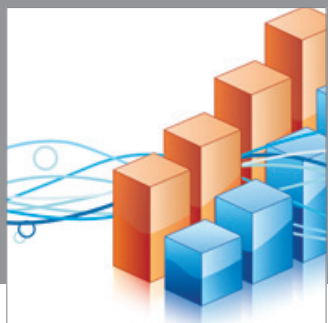

Advances in

Operations Research

mansans

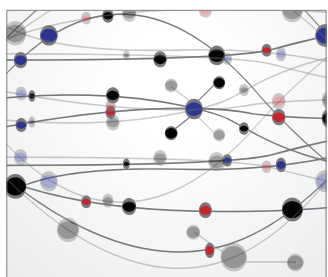

The Scientific World Journal
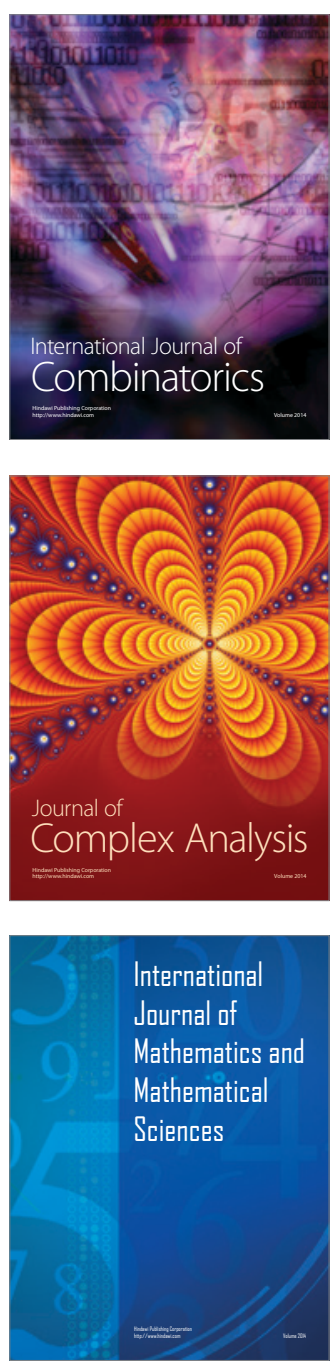
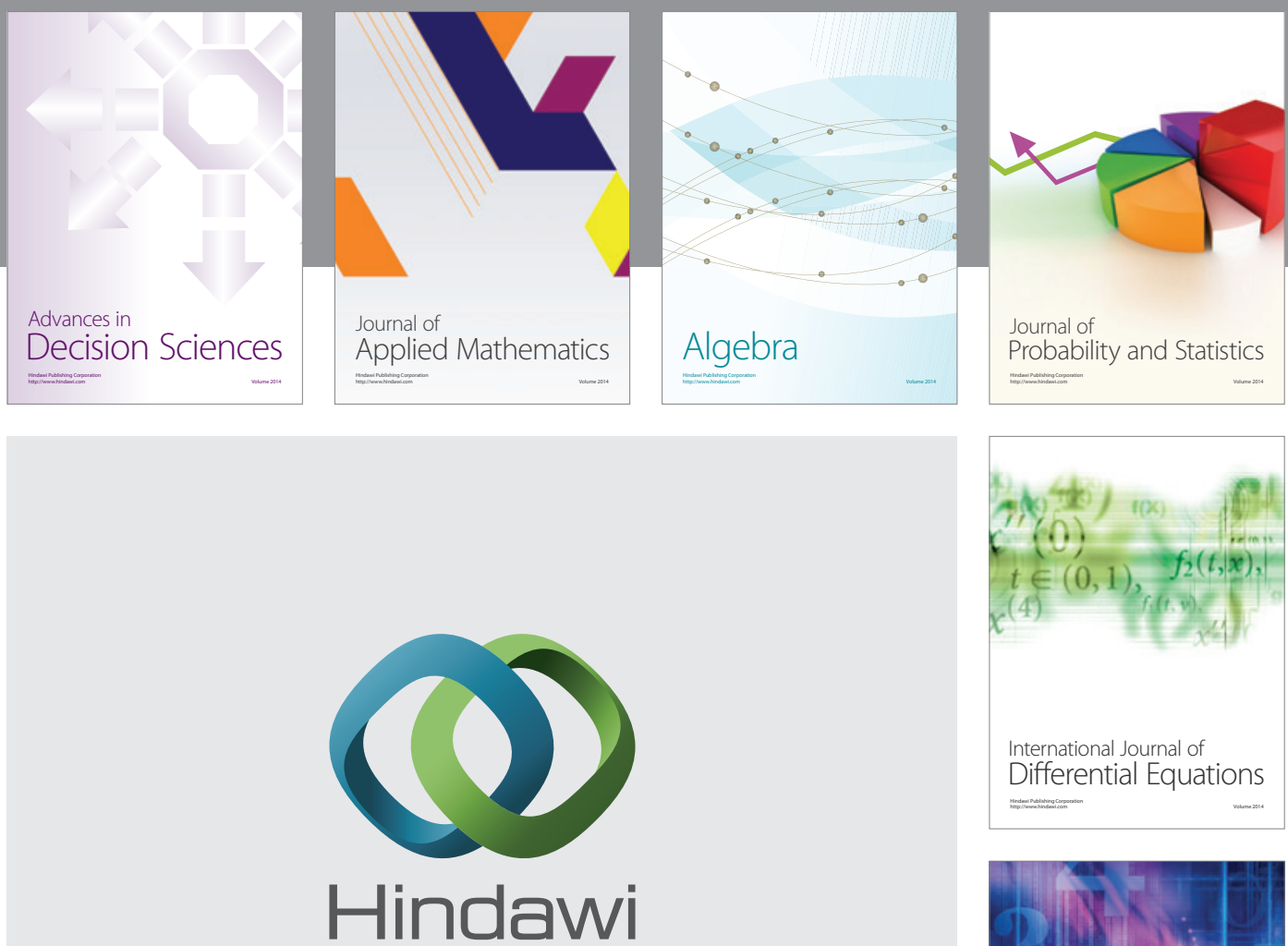

Submit your manuscripts at http://www.hindawi.com
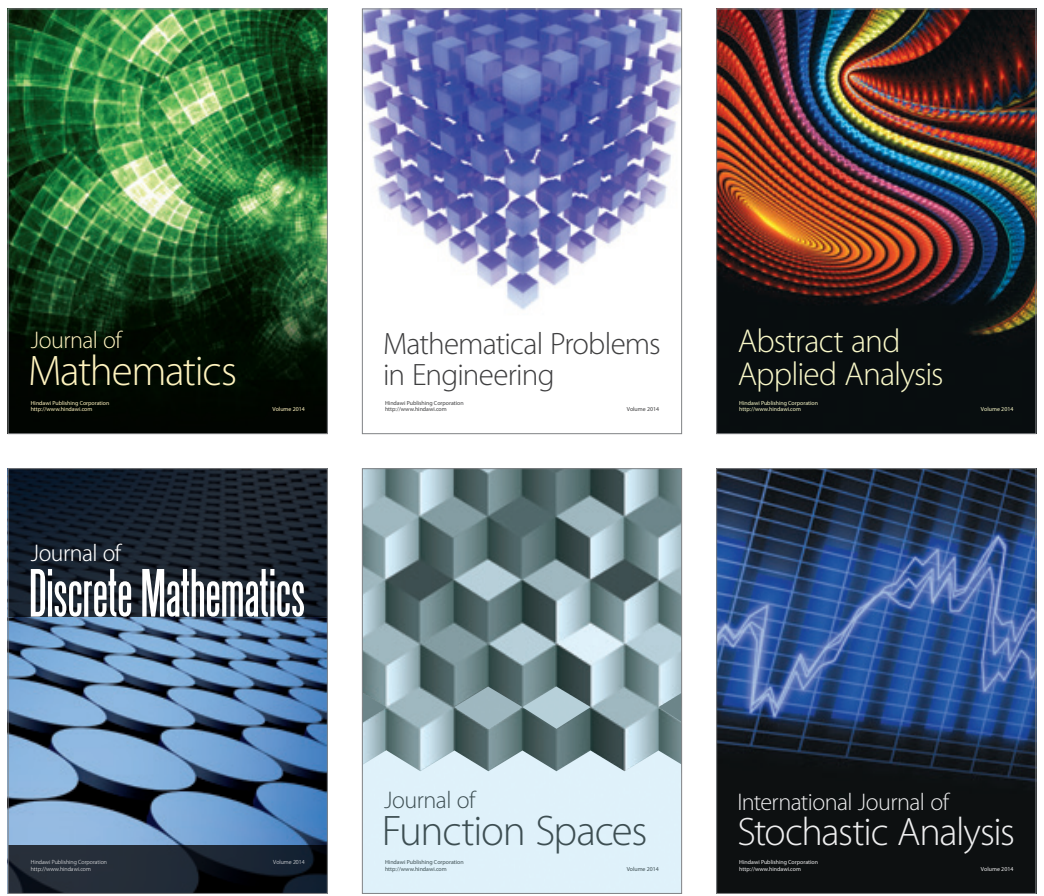

Journal of

Function Spaces

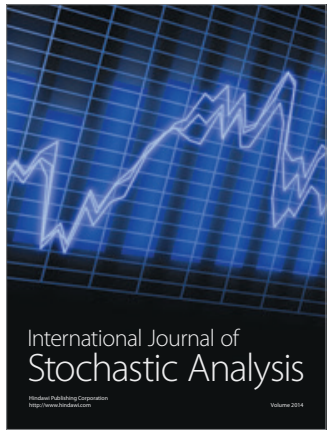

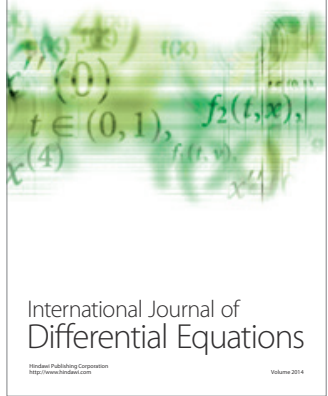
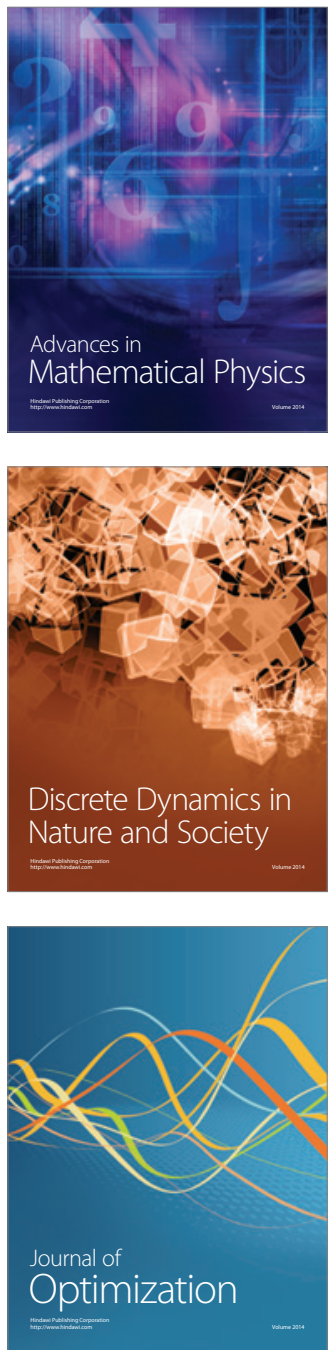